\title{
ON THE CHAIN PROBLEM OF PRIME IDEALS
}

\author{
MASAYOSHI NAGATA
}

There is a problem called the chain problem of prime ideals, which asks, when $o$ is a Noetherian local integral domain, whether the length of an arbitrary maximal chain of prime ideals in $\mathfrak{D}$ is equal to rank $\mathfrak{D}$ or not.

In the present paper, we want to show that the answer is not affirmative in the general case. On the other hand, we shall show that if the ring $D$ is quasi-unmixed (the quasi-unmixedness is a generalized notion of the unmixedness ( = equi-dimensionality)), then the answer of the above question is affirmative.

In order to discuss the problem, we first introduce some conditions on chains of prime ideals in a ring in $\S 1$. Then, in $\$ 2$, we introduce the notion of quasiunmixedness of semi-local rings and we shall show that in every quasi-unmixed semi-local ring the chain conditions which will be introduced in $\$ 1$ hold (and in particular we see that in every quasi-unmixed semi-local ring, the length of an arbitrary maximal chain of prime ideals is equal to the rank of the ring). In $\S 3$, we shall construct a counter example against the chain problem. In $\S 4$, we shall state some sufficient conditions for a Noetherian local ring to be unmixed.

Terminology. Terms which were used in Nagata [3] and [5] are used in the same sense, except for local or semi-local rings; local or semi-local rings may be non-Noetherian (see [4]).

Kesults assumed to be known. Besides some results stated in Nagata [3], we need some basic results on local rings (see, for example, $[5, \S 1]$ ) and some results contained in [5] and [6].

\section{$\S 1$. Some remarks on chains of prime ideals}

Let $\mathrm{o}$ be a ring. Then we can introduce the following condition in 0 :

The first chain condition. Every maximal chain of prime ideals in 0 is of length equal to rank 0 .

Received November 16, 1955. 
Then the following assertion holds good.

Lемма 1.1. Assume that a ring $\mathfrak{D}^{\prime}$ is integral over a ring $\mathfrak{b}$. If the first chain condition holds in $\mathrm{b}^{\prime}$, then it holds also in $\mathrm{o}$.

Proof. For a maximal chain $\mathfrak{p}_{0} \subset \mathfrak{p}_{1} \subset \ldots \subset \mathfrak{p}_{r}$ of prime ideals $\mathfrak{p}_{i}$ of $\mathfrak{b}$, there exists a chain $\mathfrak{p}_{0}^{\prime} \subset \mathfrak{p}_{1}^{\prime} \subset \ldots \subset \mathfrak{p}_{r}^{\prime}$ of prime ideals $\mathfrak{p}_{i}^{\prime}$ of $\mathfrak{o}^{\prime}$ such that $\mathfrak{p}_{i}=\mathfrak{p}_{i}^{\prime} \cap \mathfrak{o}$ for each $i$ and this last chain is maximal (see [3]). Therefore we see that $r=\operatorname{rank} \mathfrak{D}^{\prime}=$ rank $\mathrm{D}$.

On the other hand, it is obvious that

Lemma 1.2. The first chain condition holds in a ring $\mathfrak{b}$ if and only if, for every minimal prime divisor $\mathfrak{p}$ of zero of $\mathfrak{b}$, rank $\mathfrak{D}=$ rank $\mathfrak{o} / \mathfrak{p}$ and the first chain condition holds in $\mathrm{D} / \mathrm{p}$.

By these two lemmas, we see that the following condition is stronger ${ }^{1)}$ than the first chain condition:

The second chain condition. For every minimal prime divisor $\mathfrak{p}$ of zero in the ring $\mathfrak{D}$, rank $\mathfrak{D} / \mathfrak{p}=$ rank $\mathfrak{D}$ and, furthermore, the first chain condition holds in every integral extension of $\mathfrak{o} / \mathfrak{p}$.

In order to consider some other formulations of the second chain condition, we shall introduce the following two conditions for an integral domain $\mathfrak{D}$ :

Condition $\left(C^{\prime}\right)$. The first chain condition holds in every finite integral extension of $\mathfrak{b}$ contained in the derived normal ring of $\mathfrak{b}$.

Condition $\left(C^{\prime \prime}\right)$. The first chain condition holds in the derived normal ring of 0 .

By Lemma 1.1, the second chain condition (in an integral domain) implies $\left(C^{\prime \prime}\right)$ and $\left(C^{\prime \prime}\right)$ implies $\left(C^{\prime}\right)$. Conversely, we have the following two assertions.

Proposition 1 a. For an integral domain $\mathfrak{b}$, the second chain condition is equivalent to $\left(C^{\prime \prime}\right)$.

Proposition $1 \mathrm{~b}$. For a Noetherian integral domain $\mathrm{n}$, the second chain condition is equivalent to $\left(C^{\prime}\right)^{2)}$

1) It will be shown by an example in $\$ 3$ that these conditions are not equivalent to each other.

2) This can be generalized for an integral domain $v$ such that for every prime ideal $p$ of $o$ there exist only a finite number of prime ideals in the derived normal ring of which lie over $p$. 
Proof of Proposition 1 a. Let $\hat{\mathrm{o}}$ be the derived normal ring of $\hat{\mathrm{v}}$. By Lemma 1.1, we have only to show that the first chain condition holds in every integral extension of $\hat{b}$, under the assumption that the condition holds in $\hat{b}$. Since $\hat{o}$ is normal, this follows from a well known result on the chains of prime ideals in an integral extension of a normal ring (see [3]).

Proof of Proposition $1 \mathrm{~b}$. Let $\hat{p}_{0} \subset \hat{p}_{1} \subset \ldots \subset \hat{p}_{r}$ be a maximal chain of prime ideals in the derived normal ring $\hat{v}$ of $o\left(\hat{p}_{0}=0\right)$. By Lemma 1.1 and by Proposition $1 \mathrm{a}$, we have only to show that $r=$ rank $\mathrm{b}$, under the assumption that $\mathfrak{D}$ satisfies $\left(C^{\prime}\right)$. Set $\mathfrak{p}_{i}=\hat{p}_{i} \cap \mathfrak{o}$. Then there exist only a finite number of prime ideals of $\hat{v}$ which lie over $p_{i}$ (see [6]). Therefore, replacing o by a suitable finite integral extension of $\mathfrak{n}$, we may assume that each $\hat{p}_{i}$ is the unique prime ideal of $\hat{v}$ which lies over $p_{i}$. Then obviously $0=p_{0} \subset p_{1} \subset \ldots \subset p_{r}$ is a maximal chain of prime ideals in $\mathfrak{o}$ and $r=\operatorname{rank} \mathfrak{b}$.

By Proposition $1 \mathrm{a}$ and Lemma 1.1, we see that in a normal ring these four conditions are equivalent to each other. On the other hand, since the first chain condition holds in any complete Noetherian local integral domain and since every finite integral extension of a complete Noetherian local integral domain is again a complete local integral domain, we see that

Lemma 1.3. In a complete Noetherian semi-local ring $\mathfrak{b}$, the following three conditions are equivalent to each other:

(1) The validity of the first chain condition in $\mathrm{D}$.

(2) The validity of the second chain condition in $\mathrm{D}$.

(3) For any minimal prime divisor $\mathfrak{p}$ of zero, rank $\mathfrak{D} / \mathfrak{p}=\operatorname{rank} \mathfrak{D}$.

\section{§ 2. Quasi-unmixed rings}

We say that a semi-local ring is quasi-unmixed if (1) it is Noetherian and (2) the first chain condition holds in its completion (cf. Lemma 1.3). Obviously, every unmixed local ring is quasi-unmixed.

Lemma 2.1. Let o be a Noetherian semi-local ring and let $\mathrm{o}^{*}$ be its completion. If $\mathfrak{p}$ is a prime ideal of $\mathfrak{D}$ and if $\mathfrak{p}^{*}$ is a minimal prime divisor of $\mathfrak{p}^{*}$, then rank $p=$ rank $p^{*}$ (Nagata [5]).

For the proof, see [5] (though [5] treated the local case, we have immediately this generalization, because the completion of a semi-local ring 0 with maximal ideals $p_{1}, \ldots, p_{n}$ is the direct sum of completions of local rings 
$\left.\mathfrak{D}_{p_{1}}, \ldots, \mathfrak{D}_{\mathfrak{p}_{n}}\right)$.

Proposition 2. If $\mathfrak{o}$ is a quasi-unmixed semi-local ring and if $\mathfrak{p}$ is its prime ideal, then $\mathrm{v} / \mathrm{p}$ is quasi-unmixed and rank $\mathfrak{p}+$ co-rank $\mathfrak{p}=\operatorname{rank} \mathrm{o}$.

Proof. Let $\mathfrak{D}^{*}$ be the completion of $\mathfrak{D}$ and let $\mathfrak{p}^{*}$ be an arbitrary minimal prime divisor of $\mathfrak{p}^{*}$. Then rank $\mathfrak{p}=\operatorname{rank} \mathfrak{p}^{*}$ by Lemma 2.1. Since the first chain condition holds in $\mathfrak{c}^{*}$, co-rank $\mathfrak{p}^{*}=\operatorname{rank} \mathrm{D}^{*}-\operatorname{rank} \mathfrak{p}^{*}=\operatorname{rank} \mathfrak{D}-\operatorname{rank} \mathfrak{p}$, which proves our assertion because $D^{*} / \mathfrak{p}^{*}$ is the completion of $\mathfrak{o} / \mathfrak{p}$.

COROLlary. Let a be an ideal of a quasi-unmixed semi-local ring 0 . Then $\mathrm{D} / \mathrm{a}$ is quasi-unmixed if and only if the rank of every minimal prime divisor of a is equal to rank a.

THEOREM 1. If $\mathrm{o}$ is a quasi-unmixed semi-local ring, then the second chain condition holds in $\mathrm{D}^{3)}$

Proof. We see easily that the first chain condition holds in 0 by induction on rank $\mathfrak{o}$ by virtue of Proposition 2. Let $\mathfrak{p}$ be an arbitrary minimal prime divisor of zero in $\mathfrak{D}$. Then $\operatorname{rank} \mathfrak{D} / \mathfrak{p}=\operatorname{rank} \mathfrak{D}$ and $\mathfrak{D} / \mathfrak{p}$ is quasi-unmixed (Proposition 2). Therefore, in order to prove the validity of the second chain condition in $\mathfrak{b}$, it is sufficient to show that every finite integral extension $\mathfrak{b}^{\prime}$ of $\mathfrak{n} / \mathfrak{p}$ contained in the derived normal ring of $\mathfrak{o} / \mathfrak{p}$ is again quasi-unmixed. This is easily proved because the completion of $\mathfrak{b}^{\prime}$ is contained in the total quotient ring of the completion of $\mathfrak{p} / \mathfrak{p}$.

Corollary 1. If $p_{0} \subset p_{1} \subset \ldots \subset p_{r}$ is a chain of prime ideals in a quasiunmixed semi-local ring $\mathfrak{o}$ and if each $\mathfrak{p}_{i} / \mathfrak{p}_{i-1}$ is of rank 1 , then $r=$ rank $\mathfrak{p}_{r}-$ rank $p_{0}$.

Corollary 2. Let $\mathfrak{D}^{\prime}$ be an integral extension of a quasi-unmixed semi-local integral domain $\mathfrak{0}$. If $\mathfrak{p}^{\prime}$ is a prime ideal of $\mathrm{n}^{\prime}$, then rank $\mathfrak{p}^{\prime}=\operatorname{rank}\left(\mathfrak{p}^{\prime} \cap \mathfrak{o}\right) . \quad A$ chain $\mathfrak{p}_{0}^{\prime} \subset \mathfrak{p}_{1}^{\prime} \subset \ldots \subset \mathfrak{p}_{r}^{\prime}$ of prime ideals $\mathfrak{p}_{i}^{\prime}$ in $\mathfrak{b}^{\prime}$ is maximal if and only if $\left(\mathfrak{p}_{0}^{\prime} \cap \mathfrak{o}\right) \subset\left(\mathfrak{p}_{1}^{\prime} \cap \mathfrak{v}\right) \subset \ldots \subset\left(\mathfrak{p}_{r}^{\prime} \cap \mathfrak{o}\right)$ is maximal.

Remark. It will be easy to see that if $o$ is a quasi-unmixed semi-local integral domain, then every finite integral extension of $\mathfrak{o}$ is also quasi-unmixed. Further, we shall add a remark as follows: Let $\mathrm{o}$ be a Noetherian local integral

3) It was proved by Serre [7] that every regular: local ring satisfies the first chain condition. 
domain and let $\mathfrak{n}^{*}$ be its completion. Let $\mathfrak{n}^{*}$ be the radical of $\mathfrak{o}^{*}$. Then we can construct the complete semi-local ring $\left(0^{*} / n^{*}\right)[\hat{b}]$ (which has no nilpotent element), where $\hat{o}$ is the derived normal ring of $o$ (see [6]). Then, as is easily seen, there exists a natural homomorphism from the completion $\hat{\mathfrak{b}}^{*}$ of the semilocal ring $\hat{\mathfrak{v}}$ (which may not be Noetherian) onto $\left(0^{*} / \mathrm{n}^{*}\right)[\hat{\mathfrak{b}}]\left(\hat{\mathfrak{v}}^{*}\right.$ is the completion of a homomorphic image of $\hat{0}^{*}[\hat{0}]$ modulo an ideal contained in the radical of $0^{*}[\hat{o}]$ ). From this fact, if we define the notion of quasi-unmixedness without the assumption that the ring is Noetherian, then we see that every almost finite integral extension of a Noetherian quasi-unmixed semi-local integral domain is also a quasi-unmixed semi-local ring.

\section{§. Examples}

Lemma 3.1. Let o be a semi-local ring with maximal ideals $p_{1}, \ldots, p_{n}$. If every $\mathrm{D}_{i}$ is Noetherian, then $\mathrm{D}$ is also Noetherian.

Proof. Let $\mathfrak{a}$ be an ideal of $\mathrm{n}$. Since each $a \mathrm{n}_{p_{i}}$ has a finite base, there exist elements $a_{1}, \ldots, a_{s}$ of a such that $a \mathfrak{o}_{\mathfrak{p}_{i}}$ is generated by them for every $i$. Let $b$ be an element of $a$. Then there exists an element $c_{i}$ of $\mathfrak{D}$ which is not in $\eta_{i}$ such that $b c_{i}$ is in the ideal $\sum a_{j} \mathrm{D}$ for each $i$. Since $c_{i} \notin \mathfrak{p}_{i}$, all the $c_{i}$ 's generate the unit ideal $\mathbb{D}$ and we see that $b$ is in $\sum a_{j} \mathrm{n}$, which proves that $a$ has a finite base. Thus $\mathfrak{b}$ is Noetherian.

Lemma 3.2. Let $\mathfrak{o}$ be a Noetherian semi-local ring with maximal ideals $\mathfrak{p}_{1}, \ldots, \mathfrak{p}_{n}$. Assume that o contains a local ring $K$ with maximal ideal $n$ such that $\mathfrak{p}_{i} \cap K=\mathrm{n}$ and $\mathrm{n} / \mathrm{p}_{i}$ is a finite algebraic extension of $K / \mathrm{n}$. Let in be the $J$. radical of $\mathrm{D}$ and set $\xi=K+\mathrm{m}$. Then (1) 3 is a Noetherian local ring and (2) $\mathfrak{D}$ is a finite module over $\mathfrak{B}$, whence $\mathfrak{D}$ is integral over $\mathfrak{B}$.

Proof. i) It is obvious that $\xi$ is a ring. If $m \in m$, then $1 /(1+m)=1+m^{\prime}$ with an element $m^{\prime} \in m$, which shows that $m$ is the unique maximal ideal of $\xi$.

ii) Since $\mathrm{o} / \mathrm{m}$ is the direct sum of rings $\mathfrak{o} / \mathfrak{p}_{1}, \ldots, \mathfrak{o} / \mathfrak{p}_{n}$ and since each $\mathrm{n} / \mathfrak{p}_{i}$ is a finite $K / \mathfrak{n}$-module, we see that $\mathrm{o}$ is a finite $\mathrm{s}$-module, which proves (2) (see [3]).

iii) Now it is sufficient to show that $z$ is Noetherian. In order to do this, we have only to show that every prime ideal $p$ of $g$ has a finite base (see [6]; another proof will be given in Appendix at the end of this paper). Let $e_{1}$, $\ldots, e_{t}$ be a module base of 0 over $z$. Since 0 is integral over $\mathfrak{z}$, there exists 
a prime ideal $\mathfrak{p}^{\prime}$ of $\mathfrak{o}$ which lies over $\mathfrak{p}$. Then obviously $p=\mathfrak{p}^{\prime} \cap \mathrm{m}$. Therefore $\mathfrak{p}$ is an ideal of $\mathfrak{D}$ and $\mathfrak{p}$ has a finite base as an ideal of $\mathfrak{b}$; let $a_{1}, \ldots, a_{\mathfrak{u}}$ be a base of $p$ (as an ideal of $\mathfrak{D}$ ). We shall show that $p$ is generated by all the $a_{i} e_{j}$ 's. Indeed, let $b$ be an element of $p$. Then $b=\sum c_{i} a_{i}$ with $c_{i} \in \mathrm{o}$. Then $c_{i}=\sum d_{i j} e_{j}$ with $d_{i j} \in \mathfrak{g}$ and $b=\sum d_{i j} a_{i} e_{j}$. Thus $\mathfrak{p}$ has a finite base (as an ideal of $\xi$ ) and $\_$is Noetherian. Thus the lemma is proved completely.

Now we shall construct a family of Noetherian local integral domains as follows :

Let $K$ be a field and let $x$ be a transcendental element over $K$. Consider the formal power series ring $K\{x\}$ and let $z_{i}=\sum a_{i j} x^{j}\left(a_{i j} \in K ; i=1,2, \ldots, r\right.$ $(r>0))$ be algebraically independent elements over $K(x)$. Set $z_{i j}=\left(z_{i}\right.$ $\left.-\sum_{k<j} a_{i k} x^{k}\right) / x^{j-1}$. Further, let $y_{1}, \ldots, y_{m}$ (there may be none) be algebraically independent elements over $K\left[x, z_{1}, \ldots, z_{r}\right]$. Let $\mathfrak{b}_{1}$ be the ring generated by $x$ and all the $z_{i j}$ 's over $K$ (as a subring of $K\{x\}$ ) and set $\mathrm{D}_{2}=\mathrm{D}_{1}\left[y_{1}, \ldots, y_{m}\right]$.

(i) $x 0_{1}$ is a maximal ideal of $\mathfrak{b}_{1}$, because $x z_{i, j+1}=z_{i j}-a_{i j} x$ and $z_{i j} \in x 0_{1}$. Since $\left(0_{1}\right)_{x D_{1}}$ is dominated by the local ring $K\{x\}$, this is a local ring. Since the maximal ideal of $\left(D_{1}\right)_{x D_{1}}$ is principal and since this ring is a local ring, we see that this ring is Noetherian (hence this is a discrete valuation ring). Let $\mathrm{m}_{1}$ be the ideal of $\mathfrak{r}_{2}$ generated by $x, y_{1}, \ldots, y_{m}$; which is maximal and is of rank $m+1$. Further the ring $\mathfrak{v}=\left(\mathfrak{D}_{2}\right)_{\mathfrak{m}_{1}}$ is Noetherian (hence it is a regular local ring of rank $m+1$ ), because this ring is a ring of quotients of the Noetherian ring $\left(0_{1}\right)_{x_{1}}\left[y_{1}, \ldots, y_{m}\right]$.

(ii) We have obviously $0_{1}[1 / x]=K\left[x, 1 / x, z_{1}, \ldots, z_{r}\right]$. Therefore the ideal $m_{2}$ of $\mathfrak{r}_{2}$ generated by $x-1, z_{1}, \ldots, z_{r}, y_{1}, \ldots, y_{m}$ is a maximal ideal and the ring $\mathfrak{w}=\left(\mathrm{O}_{2}\right)_{\mathfrak{m}_{2}}$ is a regular local ring of rank $r+m+1$.

(iii) Set $S=\left(\mathfrak{o}_{2}-\mathfrak{m}_{1}\right) \cap\left(\mathfrak{o}_{2}-\mathfrak{m}_{2}\right)$ and $\mathfrak{o}^{\prime}=\left(\mathfrak{o}_{2}\right)_{s}$. Further set $\mathfrak{p}_{1}=\mathfrak{m}_{1} \mathfrak{o}^{\prime}$ and $\mathfrak{p}_{2}=\mathfrak{m}_{2} \mathfrak{D}^{\prime}$. Then $\mathfrak{v}=\mathfrak{v}^{\prime} \mathfrak{p}_{1}$ and $\mathfrak{w}=\mathfrak{d}_{\mathfrak{p}_{2}}^{\prime}$; they are Noetherian. Therefore $\mathfrak{D}^{\prime}$ is Noetherian by Lemma 3.1. Since $\mathfrak{b}$ and $\mathfrak{w}$ are normal rings and since $\mathfrak{b}^{\prime}=\mathfrak{v} \cap \mathfrak{w}$, we see that $\mathfrak{D}^{\prime}$ is a normal ring.

(iv) Let $m$ be the $J$-radical of $D^{\prime}$ and set $n=K+m$. Since $D^{\prime} / p_{1}=\mathfrak{o}^{\prime} / p_{2}=K$, we see that $\mathfrak{D}$ is Noetherian and $\mathfrak{v}^{\prime}$ is the derived normal ring of $\mathfrak{D}$ by Lemma 3. 2.

Now we want to explain some meanings of the example 0 which we have constructed. 
(I) The second chain condition is not satisfied by 0 .

Proof. The derived normal ring $\mathfrak{b}^{\prime}$ of $\mathfrak{b}$ has two maximal ideals; one is of rank $m+1$ and the other is of rank $r+m+1$.

Consider the special case where $m=0$. Then since $p_{1}$ is of rank 1 , there exists a one to one correspondence between prime ideals of $\mathfrak{w}$ and those of $\mathfrak{v}$ such that $q^{\prime}$ corresponds to $\mathfrak{q}$ if and only if $q^{\prime} \cap 0=q$. Since $\mathfrak{w}$ is regular, the first chain condition is satisfied by $\mathfrak{w}$ by virtue of Theorem 1 , which shows the validity of the first chain condition in $\mathrm{n}$ (in this special case). Thus we have

(II) When $m=0$, the ring $n$ is a Noetherian local integral domain in which the first chain condition holds but not the second one.

(III) If $m$ is positive, then the ring o does not satisfy the first chain condition. Therefore, in this case, $\mathrm{D}$ is a counter example against the chain froblem of prime ideals.

Proof. Set $q=x \mathrm{u}^{\prime} \cap \mathrm{o}_{\text {. Th }}$ Then obviously $q=x 0^{\prime} \cap p_{2}$. Therefore $q^{\prime} p_{2}=p_{2} g^{\prime} p_{2}$ (because $x \notin y_{2}$ ), which shows that there is no prime ideal of $\mathfrak{b}^{\prime}$ contained in $f_{2}$ which lies over $q$ (because, since $m>0, x 0^{\prime}$ is not a maximal ideal of $v^{\prime}$ and $q$ is not a maximal ideal of 0 ). Therefore we see that $0^{\prime} x^{\prime}{ }^{\prime}$ is the derived normal ring of $o_{q}$ and rank $q=$ rank $x \mathcal{D}^{\prime}=1$. Now consider any maximal chain of prime ideals of $\mathfrak{D}$ which goes through $q=q_{1}$, say, $0 \subset q_{1} \subset q_{2} \subset \ldots \subset q_{v}$. Let $0 \subset q_{1}^{\prime} \subset q_{2}^{\prime} \subset \ldots \subset q_{v}^{\prime}$ be a chain of prime ideals of $v^{\prime}$ such that $q_{i} \cap 0=q_{i}$ for each $i$. Then $q_{1}^{\prime}=x 0^{\prime}$ and therefore each $q_{i}^{\prime}$ is contained in $p_{1}$. Since rank $p_{1}$ $=m+1$, we have $v \leqq m+1<$ rank $\mathfrak{o}(=r+m+1)$. Thus the proof is completed.

(IV) The multiplicity of $\mathrm{o}$ is one. Thus $\mathrm{o}$ is an example of Noetherian local integral domain of multiplicity one which is not regular.

Proof. Since $\mathrm{o}^{\prime} / \mathrm{p}_{2}=\mathrm{o} / \mathrm{m}=K$ and since $\mathrm{p}_{2}$ is the unique prime ideal of $\mathrm{b}^{\prime}$ such that its rank is equal to that of 0 , we have $e(m)=r m\left(m 0^{\prime} ; 0\right)=e\left(m 0^{\prime} p_{2}\right)$ (see [5]). Since $m_{0_{1}}^{\prime} \mathfrak{p}_{2}=\mathfrak{n}_{2} 0_{\mathfrak{p}_{2}}^{\prime}$ and since $\mathrm{o}_{\mathfrak{p}_{2}}^{\prime}$ is a regular local ring, we see that the multiplicity of 0 is equal to one.

(V) $\mathcal{D}$ is not a homomorphic image of any quasi-unmixed local ring.

Proof. $\mathfrak{o}$ is not quasi-unmixed by (I) above. Therefore we see the assertion 
by Theorem 1 .

Remark. There is a maximal chain $0 \subset p_{1}^{\prime} \subset \ldots \subset p_{m+1}^{\prime}=p_{1}$ of prime ideals in $\mathfrak{D}^{\prime}$ such that $0 \subset\left(\mathfrak{p}_{1}^{\prime} \cap \mathfrak{D}\right) \subset \ldots \subset\left(\mathfrak{p}_{m+1}^{\prime} \cap \mathfrak{D}\right)=\mathrm{m}$ is not a maximal chain of prime ideals in $\mathfrak{D}$.

Proof. Let $\mathfrak{p}_{m}^{\prime}$ be the ideal of $\mathfrak{b}^{\prime}$ generated by $y_{1}, \ldots, y_{m}$; it is a prime ideal of rank $m$ and contained in $m$ (hence $\mathfrak{p}_{m}^{\prime}$ is a prime ideal of $\mathfrak{b}$ ). Obviously $\mathfrak{p}_{1} / p_{m}^{\prime}$ is of rank 1 and $m / p_{m}^{\prime}$ is of rank $r+1$, which proves our assertion.

\section{$\S$ 4. Some sufficient conditions for a local ring to be unmixed}

As is well known, geometric local rings, spots and regular local rings are unmixed. More generally we can prove

TheOREm 2. Assume that a Noetherian local ring $\mathrm{D}$ has a distinct sysiem of parameters. ${ }^{4)}$ Then, for an ideal a of $\mathfrak{b}, \mathfrak{o} / \mathfrak{a}$ is uninixed if and only if the rank of every prime divisor $p$ of a is equal to rank a.

The only if part of this theorem is obvious. In order to prove the if part, we shall prove some preliminary results:

Lemma 4.1. Let $\mathfrak{D}$ be a Noetherian local ring. Assume that the zero ideal of $\mathfrak{D}$ is a primary ideal with prime divisor $\mathfrak{p}$. If $\mathfrak{D} / \mathfrak{p}$ is unmixed, then $\mathfrak{D}$ is also unmixed.

Proof. As was shown by [5], any prime divisor of zero in the completion $\mathfrak{o}^{*}$ of $\mathrm{o}$ is contained in a prime divisor of $\mathrm{po}^{*}$, from which our assertion follows immediately.

Lemma 4.2. Assume that $\mathrm{o}$ is an unmixed local ring. If $\mathrm{p}$ is a prime divisor of zero, then $\mathrm{o} / \mathrm{p}$ is unmixed.

Proof. Let $a$ be a nonzero element of $0: \mathfrak{p}$. Then $0: a \mathfrak{0}=\mathfrak{p}$. On the other hand, let $0^{*}$ be the completion of 0 and let $b^{*}$ be an element of $0^{*}$ which is not a zero-divisor. Let $c^{*}$ be an element of $0^{*}$ such that $b^{*} c^{*} \in \mathfrak{p o}$. Since $0: a 0=p$, we have $0: a 0^{*}=\mathrm{po}^{*}$. Since $b^{*} c^{*} a=0$ and since $b^{*}$ is not a zero-divisor, we have $c^{*} a=0$ and $c^{*} \in 0: a 0^{*}=p 0^{*}$ and $b^{*}$ is not a zero-divisor modulo po*, which shows that $\mathfrak{o} / \mathfrak{p}$ is unmixed.

4) As for the notion of distinct system of parameters, see [5]. 
Remark. The above proof shows, without the assumption of unmixedness, that any prime divisor of $\mathfrak{p o}^{*}(\mathfrak{p}$ being a prime divisor of zero in $\mathfrak{D}$ as in Lemma 4.1 ) is contained in a prime divisor of zero in $D^{*}$.

Combining these two lemmas, we have

Proposition 3. A Noetherian local ring $\mathfrak{D}$ is unmixed if and only if for every prime divisor $\mathfrak{p}$ of zero (1) rank $\mathfrak{o} / \mathfrak{p}=$ rank $\mathfrak{o}$ and (2) $\mathfrak{o} / \mathfrak{p}$ is unmixed.

Now we shall prove Theorem 2. By Proposition 3, we may assume that $\mathfrak{a}=\mathfrak{p}$. Let $a_{1}, \ldots, a_{r}(r=\operatorname{rank} p)$ be elements of $p$ such that $\operatorname{rank}\left(\sum a_{i} \mathrm{D}\right)=r$. Then $\mathrm{o} /\left(\sum a_{i} 0\right)$ has a distinct system of parameters and is unmixed (see [5]). Since $p$ is a minimal prime divisor of $\sum a_{i}$, we see that $\mathfrak{o} / \mathfrak{p}$ is unmixed by Lemma 4.2 (or Proposition 3). Thus Theorem 2 is proved.

Proposition 4. Assume that a class $G$ of a Noetherian local rings satisfies the following 4 conditions. Then every member of $G$ is unmixed and analytically unramified.

(1) If $\mathfrak{D} \in G$, then o contains no nilpotent element.

(2) If $\mathfrak{D} \in G$ and if $\mathfrak{p}$ is a prime ideal of $\mathfrak{D}$, then $\mathfrak{v} / \mathfrak{p} \in G$.

(3) If an integral domain $\mathfrak{D}$ is in $G$, then the derived normal ring $\mathfrak{D}^{\prime}$ of $\mathfrak{D}$ is a finite $\mathrm{D}$-module and for every maximal ideal $\mathrm{m}^{\prime}$ of $\mathrm{D}^{\prime} \mathrm{D}^{\prime} \mathrm{m}$, is in $G$.

(4) If $\mathfrak{v} \in G$, then the second chain condition holds in $\mathfrak{0}$.

Proof. By (2), we can make use of the induction argument on the rank of a member $\mathfrak{D}$ of $G$. On the other hand, by (2) and (4) we may treat only the case where $\mathfrak{D}$ is an integral domain. Then by (3) and (4) we may assume that $\mathfrak{D}$ is a normal ring. Therefore, the proof of analytical unramifiedness due to Zariski [8] (see [2] or [4]) can be applied and we see easily our assertion.

COROLlaRY. If a Noetherian local ring 0 is a ring of quotients of a complete Noetherian local integral domain, then $\mathfrak{D}$ is unmixed and analytically unramified.

Proposition 5. Assume that a Noetherian local ring 0 is a subspace of an unmixed local ring $\mathrm{n}^{\prime}$. Assume further that 1) rank $\mathrm{o}=$ rank $\mathrm{b}^{\prime}$ and 2) the maximal ideal $\mathrm{m}$ of o generates a primary ideal belonging to the maximal ideal $\mathrm{m}^{\prime}$ of $\mathrm{b}^{\prime}$. Then $\mathrm{o}$ is also unmixed.

Proof. Let $\mathfrak{D}^{*}$ and $\mathfrak{D}^{\prime *}$ be the completions of $\mathfrak{D}$ and $\mathfrak{D}^{\prime}$ resepctively and let 
$\mathfrak{p}^{*}$ be a prime divisor of zero in $\mathfrak{D}^{*}$. Since $\mathfrak{D}$ is a subspace of $\mathfrak{D}^{\prime}, \mathfrak{D}^{*}$ is a subring of $\mathfrak{b}^{\prime *}$. Therefore there exists a prime divisor $\mathfrak{p}^{\prime *}$ of zero in $\mathfrak{D}^{\prime *}$ which contains $\mathfrak{p}^{*}$. Starting from $\mathfrak{p}_{0}^{\prime *}=\mathfrak{p}^{* *}$, we construct a maximal chain of prime ideals $\mathfrak{p}_{i}^{\prime *}$ as follows: When $p_{i-1}^{*}(i \leqq$ rank $n)$ is defined, let $a$ be an element of $m$ which is not in $\mathfrak{p}_{i-1}^{\prime *}$ and let $\mathfrak{p}_{i}^{\prime *}$ be a minimal prime divisor of $\mathfrak{p}_{i-1}^{\prime *}+\boldsymbol{a}^{\prime *}$. Set $\mathfrak{p}_{i}^{*}$ $=\mathfrak{p}_{i}^{\prime *} \cap \mathfrak{o}^{*} . \quad$ By our construction, $p^{*} \subseteq p_{0}^{*} \subset p_{1}^{*} \subset \ldots \subset p_{r}^{*}=\mathfrak{m} \quad(r=\operatorname{rank} \mathfrak{o})$ $\left(p_{i-1}^{*} \neq p_{i}^{*}\right.$ because $a \in p_{i}^{*}$ and $\left.a \notin p_{i-1}^{*}\right)$. Therefore, co-rank $p^{*} \geqslant$ rank $\mathfrak{0}$ and co-rank $\mathfrak{p}^{*}=$ rank $\mathfrak{D}$. Thus $\mathfrak{D}$ is unmixed.

Proposition 6. If a local ring $\mathrm{D}$ is unmixed, then for every prime ideal $\mathrm{p}$ of $\mathrm{o}$ the ring $\mathrm{O}_{\mathfrak{p}}$ is also unmixed.

Proof. Let $\mathfrak{b}^{*}$ be the completion of $\mathfrak{D}$ and let $\mathfrak{p}^{*}$ be a minimal prime divisor of $\mathfrak{p o}^{*}$. Then, for every primary ideal $q$ of $\mathfrak{D}$ belonging to $p, q 0^{*} p^{*} \cap \mathfrak{o}_{\mathfrak{p}}=q \mathbb{D}_{\mathfrak{p}}$; for, let $a$ be an element of $q 0^{*}{ }_{p^{*}} \cap 0$. Then $q 0^{*}: a 0^{*}=(q: a 0) 0^{*}$ is not contained in $p^{*}$, and it follows that $\mathfrak{q}: a 0=\mathfrak{p}$ and $a \in q$. Therefore we see that $\mathrm{p}_{\mathfrak{p}}$ is a subspace of $0^{*} p^{*}$. By the corollary to Proposition 4 , we see that $0^{*} p^{*}$ is unmixed by virtue of Proposition 3. Now we see that $o_{p}$ is unmixed by Proposition 5.

Remark. In a similar way, we can prove that if a local ring 0 is quasiunmixed and if $p$ is a prime ideal, then $\mathfrak{o}_{\mathfrak{p}}$ is quasi-unmixed.

Proposition 7. Let $\mathrm{o}$ be an unmixed local integral domain and let $x$ be an element of a field containing $\mathrm{b}$. Then for every prime ideal $\mathfrak{p}$ of $\mathrm{o}[x]$, the local ring $\mathrm{D}[x]_{\mathfrak{p}}$ is unmixed.

Proof. Since $\mathfrak{D}_{(\mathfrak{p} \cap \mathrm{D})}$ is unmixed by Proposition 6, we may assume that $\mathfrak{p}$ contains the maximal ideal $m$ of $p$. On the other hand, we may assume that $p$ is maximal by Proposition 6 .

(i) When $x$ is integral over $\mathfrak{D}$, then the assertion is easily proved and we assume that $x$ is not integral over $n$.

(ii) When $x$ is algebraic over $\mathrm{D}$, we take an element $a(\neq 0)$ of $\mathrm{D}$ such that $a x$ is integral over $\mathfrak{D}$. Then $\mathfrak{n}[a x]_{\mathfrak{p}^{\prime}}$ is unmixed, where $\mathfrak{p}^{\prime}=\mathfrak{p} \cap \mathrm{D}[a x]$. Therefore we may assume that either $x$ is in the field of quotients of $D$ or transcendental over $\mathfrak{D}$. In the former case, there exists a transcendental element $z$ over $\mathfrak{D}$ and a prime ideal $\mathfrak{q}$ of $\mathfrak{D}[z]$ such that $\mathfrak{D}[x]=\mathfrak{D}[z] / q$ and $q$ is of the form $q=(a z$ $+b) \mathrm{D}[1 / a, z] \cap \mathrm{D}[z]$. Thus, assuming that $z=x$, we have only to prove that 
both $\mathfrak{D}[z]_{\mathfrak{p}}$ and $\mathfrak{D}[z]_{\mathfrak{p}} / \mathfrak{q}[z]_{\mathfrak{p}}$ are unmixed.

(iii) Since we assumed that $p$ is maximal, there exists a monic polynomial $f(z)$ over $\mathfrak{o}$ such that $p$ is generated by $m$ and $f(z)$. Observe here that $f(z)$ is irreducible modulo $\mathrm{m}$.

(iv) Let $0^{*}$ be the completion of $o$ and let $p^{*}$ be the maximal ideal of $0^{*}[z]$ generated by $p$. Then $D^{*}[z]_{p^{*}}$ contains $0[z]_{p}$ as a dense subspace. Since $0^{*}[z]_{p^{*}}$ is a homomorphic image of a regular local ring and since $\mathfrak{D}^{*}$ is unmixed, we see that $\mathfrak{D}^{*}[z]_{\mathfrak{p}^{*}}$ is unmixed by Theorem 2 , which proves that $[z]_{\mathfrak{p}}$ is unmixed. Now we shall consider $\mathfrak{D}[z]_{\mathfrak{p}} / \mathfrak{q}[z]_{\mathfrak{p}}$; this is a dense subspace of $\mathfrak{v}^{* *}=$ $\mathfrak{D}^{*}[z]_{\mathfrak{p}^{*}} / q \mathrm{O}^{*}[z]_{\mathfrak{p}^{*}}$ (because $[z]_{\mathfrak{p}}$ is a dense subspace of $\mathfrak{D}^{*}[z]_{\mathfrak{p}^{*}}$ ). $\mathrm{D}^{* *}$ is a homomorphic image of a regular local ring. Since $a$ is not a zero-divisor in $0^{* *}$, $\mathrm{qD}^{*}[z]_{\mathrm{p}^{*}}$ is equal to $\left((a z+b)_{\mathrm{D}^{*}}[1 / a, z] \cap \mathrm{D}^{*}[z]\right) \mathrm{D}^{*}[z]_{\mathrm{p}^{*}}$ and every prime divisor of this ideal is of rank 1 . From this and from the fact that $D^{* *}$ is a homomorphic image of a regular local ring, we see that $0^{* *}$ is unmixed and $\mathfrak{o}[z]_{\mathfrak{p}} / \mathrm{q}[z]_{\mathfrak{p}}$ is also unmixed. Thus the proof is cornpleted.

Corollary. Assume that $\mathcal{D}$ is an unmixed local integral domain and let be a finitely generated integral domain over $\mathrm{b}$. Then for every prime ideal po of $\mathfrak{g}$, the ring $\mathfrak{a p}_{\mathfrak{p}}$ is unmixed.

Remark. Assume that a Noetherian local integral domain 0 is analytically

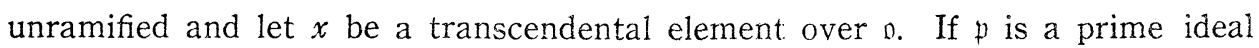
of $o[x]$ which contains the maximal ideal $m$ of $\mathfrak{o}$, then $o[x]_{\mathfrak{p}}$ is analytically irreducible.

Proof. Let $\mathfrak{p}^{*}$ be the completion of $\mathfrak{v}$ and let $p^{*}$ be the ideal $\mathfrak{p o}^{*}[x]$, which is prime. Then $\mathfrak{D}^{*}[x]_{p^{*}}$ contains $\mathfrak{D}[x]_{\mathfrak{p}}$ as a dense subspace (which can be proved easily, separating into two cases; the case $p=m 1[x]$ and the other case). Thus we may assume that 0 is complete. Since the derived normal ring of $\mathfrak{o}$ is a local ring and is a finite 0 -module, we may assume that 0 is normal. (i) When $p$ is maximal, let $f(x)$ be a monic polynomial such that $p$ is generated by $m$ and $f(x)$. Then $o[x]_{p} / f(x)_{0}[x]_{p}$ is a finite integral extension of $\mathrm{o}$ and is a complete local integral domain. Then the method of Zariski used in the proof of Proposition 4 can be applied and we see that $\mathfrak{o}[x]_{p}$ is analytically irreducible. (ii) When $\mathfrak{p}=\operatorname{mo}[x]$, let $\mathfrak{r}$ be an unramified regular local ring contained in 0 such that $\mathfrak{D}$ is a finite $r$-module. If $\mathfrak{o}$ is separable over $\mathfrak{r}$, then the method in Zariski 
[9] (see also [4]; cf. [2] and [2, II]) can be applied and we see that the completion of $\mathfrak{o}[x]_{\mathfrak{p}}=\mathfrak{o}(x)$ is a normal ring. In the general case, let $\mathfrak{x}^{\prime}$ be an integral extension of $\mathfrak{r}$ contained in $\mathfrak{D}$ such that $\mathfrak{r}^{\prime}$ is separable over $\mathfrak{r}$ and $\mathfrak{D}$ is purely inseparable over $\mathfrak{r}^{\prime}, \mathfrak{r}^{\prime}$ being a normal local ring. Set $\mathfrak{p}^{\prime}=\mathfrak{p} \cap \mathfrak{r}^{\prime}[x]$. Then the completion of $\mathfrak{r}^{\prime}[x]_{p^{\prime}}$ is a normal ring. Therefore we see that the zero ideal of the completion of $\mathfrak{o}(x)$ is a primary ideal. As was shown by [5], the ring $\mathfrak{D}(x)$ is analytically unramified. Therefore we see that $\mathfrak{o}(x)$ is analytically irreducible.

\section{§ 5. Some related open questions}

Problem 1. The writer does not know any example of Noetherian local integral domain such that the zero ideal of its completion has imbedded prime divisors. Is there such an example or not? In particular; is there a quasiunmixed local integral domain which is not unmixed?

Problem 2. Let $\mathfrak{p}$ be a prime ideal of an unmixed local ring $\mathfrak{b}$. Then: Is $\mathfrak{o} / \mathfrak{p}$ necessarily unmixed?

Problem 3. Let $\mathfrak{h}$ be a Henselian Noetherian local integral domain. Then : Does $\mathfrak{h}$ satisfy the first chain condition?

Remark. This Problem 3 is equivalent to each of the following two problems :

Problem 3 . Let $\mathfrak{h}$ be a Henselian Noetherian local integral domain. Then : Does $\mathfrak{h}$ satisfy the second chain condition?

Problem 3". Let $\mathfrak{v}$ be a Noetherian local integral domain. Assume that the derived normal ring of $\mathfrak{o}$ has only one maximal ideal. Then: Does $\mathfrak{D}$ satisfy the first (or the second) chain condition?

Problem 4. Let $\mathfrak{h}$ be a Henselian Noetherian local integral domain and let $a$ be an element of the field of quotients of $\mathfrak{h}$ such that neither $a$ nor $a^{-1}$ is integral over $\mathfrak{h}$ and that for the maximal ideal $m$ of $\mathfrak{h}, m \mathfrak{h}[a]$ is a prime ideal. Set $\mathfrak{o}=\mathfrak{h}[a] \operatorname{mb}[a]$. Then rank $\mathfrak{v}=\operatorname{rank} \mathfrak{h}-1$. Let $\mathfrak{p}$ be a maximal ideal of the derived normal ring of $\mathfrak{b}$. Then: Is the rank of $\mathfrak{p}$ equal to rank $\mathfrak{b}$ ?

Remark. If we should have an affirmative answer for Problem 4, then we would have also an affirmative answer for Problem 3. 


\section{Appendix. A proof of a theorem due to Cohen}

The following theorem was proved by Cohen making use of the fact that every submodule of a finite module over a Noetherian ring is also finite (see [6]):

THEOREM. Let $\mathrm{o}$ be a ring. If every prime ideal of $\mathrm{o}$ has a finite base, then 0 is Noetherian.

Here we shall give another proof of this theorem making use of the following

Lemma. Let $a$ be an ideal of $a$ ring $\mathfrak{0}$ and let $b$ be an element of $\mathfrak{b}$. If both $a+b o$ and $a: b 0$ have finite bases, then so does $a$, too. (Oka [1])

Proof. There exist a finite number of elements $a_{1}, \ldots, a_{n}$ of $a$ such that $a+b s$ is generated by $a_{1}, \ldots, a_{n}, b$. On the other hand, let $c_{1}, \ldots, c_{m}$ be a base of $a: b$. Let $a^{\prime}$ be the ideal generated by $a_{1}, \ldots, a_{n}, c_{1} b, \ldots, c_{m} b$. Obviously $a^{\prime} \leqq a$. Let $x$ be an element of $a$. Then $x=\sum y_{i} a_{i}+z b$ with $y_{i}, z \in 0$. Since $z b \in \mathfrak{a}, z \in \mathfrak{a}: b$ ) and $z b \in b(\mathfrak{a}: b 0)$. Thus we see that $x \in \mathfrak{a}^{\prime}$ and $\mathfrak{a}=\mathfrak{a}^{\prime}$.

Proof of the theorem. Assuming the contrary, let $F$ be the set of ideals of 0 which have no finite bases. Then $F$ is an inductive set and $F$ contains a maximal member $a$. Then $a$ is not prime by our assumption. Therefore there are elements $b, c \in \mathcal{D}$ which are not in a such that $b c \in \mathfrak{a}$. Then $\mathfrak{a}+b \mathfrak{0}$ and $\mathfrak{a}: b \mathfrak{a}$ are different from $\mathfrak{a}$ and have finite bases by the maximality of $\mathfrak{a}$, which shows that a has a finite base by the lemma and is a contradiction. Thus the theorem is proved.

\section{BiBLIOGRAPHY}

[1] K. Oka, Sur les fonctions analytiques de plusieurs variables, VIII-Lemme fondamental, J. of Math. Soc. of Japan, vol. 3 (1951), pp. 204-214.

[2] M. Nagata, Some remarks on local rings, Nagoya Math. J., vol. 6 (1953), pp. 53-58.

[2, II] M. Nagata, Some remarks on local rings, II, Memoirs Kyoto Univ., ser. A, vol. 28, No. 2 (1954), pp. 109-120.

[3] M. Nagata, Basic theorems on general commutative rings, Memoirs Kyoto Univ., ser. A, vol. 29, No. 1 (1955), pp. 59-77.

[4] M. Nagata, A general theory of algebraic geometry over Dedekind domains, I, Amer. J. of Math., vol. 78, No. 1 (1956), pp. 78-116.

[5] M. Nagata, The theory of multiplicity in general local rings, forthcoming.

[6] M. Nagata, The derived normal rings of Noetherian integral domains, Memoirs Kyoto Univ., ser. A, vol. 29, No. 3 (1955), pp. 293-303. 
[7] J. P. Serre, Sur la dimension homologique des anneaux et des modules noethériens, forthcoming.

[8] O. Zariski, Analytical irreducibility of normal varieties, Ann. of Math., vol. 49 (1948), pp. 352-361.

[9] O. Zariski, Sur la normalité analytique des variété normales, Ann. L'inst. Fourier, vol. 2 (1950), pp. 161-164.

Mathematical Institute

Kyoto University 